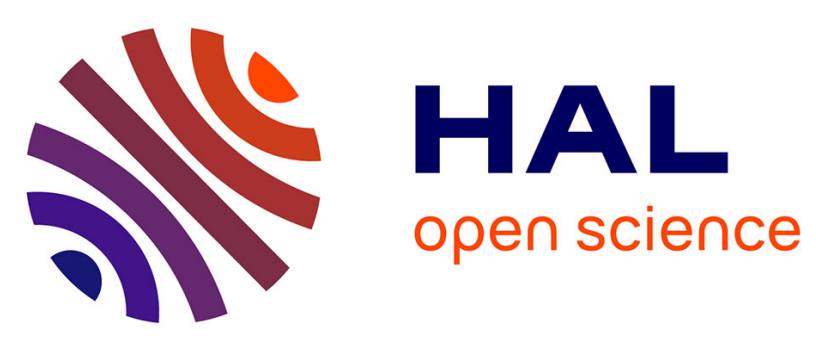

\title{
A Multi-perspective and Multi-level Based Approach to Organize and Represent Inventive Knowledge
}

\author{
Longfan Liu, Denis Cavallucci, Yan Li
}

\section{To cite this version:}

Longfan Liu, Denis Cavallucci, Yan Li. A Multi-perspective and Multi-level Based Approach to Organize and Represent Inventive Knowledge. 19th International TRIZ Future Conference (TFC), Oct 2019, Marrakesh, Morocco. pp.228-239, 10.1007/978-3-030-32497-1_19 . hal-02905525

\section{HAL Id: hal-02905525 \\ https://hal.inria.fr/hal-02905525}

Submitted on 23 Jul 2020

HAL is a multi-disciplinary open access archive for the deposit and dissemination of scientific research documents, whether they are published or not. The documents may come from teaching and research institutions in France or abroad, or from public or private research centers.
L'archive ouverte pluridisciplinaire HAL, est destinée au dépôt et à la diffusion de documents scientifiques de niveau recherche, publiés ou non, émanant des établissements d'enseignement et de recherche français ou étrangers, des laboratoires publics ou privés. 


\title{
A multi-perspective and multi-level based approach to organize and represent inventive knowledge
}

\author{
LIU Longfan ${ }^{1,2}$, Denis CAVALLUCI ${ }^{2}$ and LI Yan ${ }^{1}$ \\ 1 School of Manufacturing Science and Engineering, Sichuan University, NanYihuan \\ Road No.24, Chengdu 610065, Sichuan Province, China. \\ 2 ICube (UMR-CNRS 7357), 24 Boulevard de la Victoire, 67000, Strasbourg, France.
}

\begin{abstract}
Through analyzing the solving mechanism of product inventive design problem based on TRIZ theory, we propose in this paper a multi-perspective and multi-level knowledge organization model, so as to fill the "GAP" between the general solution and the particular solution in the process of solving an inventive problem. We labeled here design knowledge by five attributes, i.e., TRIZ, function, behavior-flow, effect, and domain, each of which includes respectively three levels that are conceptual level, semantic level and factual resource level. Thereby, a multi-perspective and multi-level local knowledge base is established. Besides, we put forward also a card-type knowledge representation template, which divides the traditional cases, patents and other knowledge into several independent parts, and each of them can be displayed and pushed to designers in form of cards. Thus, it's conductive to stimulate designers' analogy and association. One multi-perspective and multi-level knowledge organization based inventive problem solving model is conclusively established, which supports analysis and problem-solving in different perspectives and at different levels. A conceptual design of an ultrasonic washing machine is illustrated at the end as an implemented example for this proposed model.
\end{abstract}

Keywords: Knowledge representation, inventive problem solving, TRIZ

\section{Introduction}

Product design is a continuous and purposive kind of creative thinking activity, which is carried out to meet certain needs of knowledge ${ }^{[1]}$. From the cognitive perspective, the process of solving the design problem can be regarded as that of "the correlation mining between the problem and the existing knowledge, as well as the knowledge transfer and combination" ${ }^{[2]}$. The knowledge requirement runs through the entire life cycle of product design.

Bringing knowledge forward and making it explicit is one of the key roles of knowledge representation ${ }^{[3]}$. Recent studies about the organization and representation are based on artificial intelligence, which focuses on the computer processing of knowledge. All the presentations and organizations of knowledge are structural, which the computer can handle and be regarded as a set of structural data and a set of correlative operation. Cong et al. ${ }^{[4]}$ and Paul et al. ${ }^{[5]}$ put forward a method of patent knowledge classification based on inventive principles, as well as assisted by ontological reasoning technique. And it can also automatically provide the relevant patent knowledge to designer during the solving process of TRIZ problem. Yu et al. ${ }^{[6]}$ and Park et al. ${ }^{[7]}$ proposed to analyze and retrieve the patent items with the help of the trend of evolution so as to solve the current problem. Zhang et al. ${ }^{[8]}$ proposed a semantic ontology 
based knowledge representation model for invention principles. Qin et al. ${ }^{[9]}$ proposed a Requirement-Function-Behavior-Structure-Evolution (RFBSE) model to obtain useful knowledge and experience in the design process. There are also some innovative design knowledge management platforms including Aulive ${ }^{[10]}$, Pro/Innovator ${ }^{[11]}$, PIXSEB (Driving innovation through the semantic extraction of patent content) ${ }^{[12]}$, Creap (Creativity Design Service Platform) ${ }^{[13]}$, CREAX ${ }^{[14]}$ etc. However, the former research in knowledge representation model emphasized chiefly the description of connection between design objects and design processes, and nevertheless lacked an efficient systematic approach that can integrate the design object, problem-solving process, as well as recognition strategies.

Inventive design is people-centered, and people should be finally engaged in this design process. Knowledge must be oriented to comprehension and motivation for humans. Knowledge representation should focus on human's reading, understanding, association. However, the kind of knowledge presented to people is rather important. The main role of computers is to organize, and to represent knowledge. In fact, it is a state in which human-machine forms a joint cognition, i.e., the combination of artificial intelligence and human cognition forms a cognitive channel that is conducive to design innovation. Therefore, the organization and representation of knowledge must satisfy ${ }^{[15]}$ : (I) It should be benefit of the designer's perception. (II) It should be in favor of the designer's association analogy and knowledge transfer. (III) It should make for computer retrieval and push forward. (IV) It should avail the expansion of knowledge and the use of resources.

Besides, the design problem is typical ill-structural problem ${ }^{[16]}$. The design problem solving process is a process of clarifying and refining the initial problem, along with repeated iterations in different abstraction level, i.e. designers may need the knowledge of different abstraction levels at different time to solve the problems. Knowledge organization and representation must include different abstraction levels of knowledge to assist designers to obtain design information of relevant level, which facilitates thereby the analysis and problem-solving from different conceptual levels and different perspectives.

\section{The Mechanism of creative problem solving based on multi- perspective and multi-level}

TRIZ (Theory of Inventive Problem Solving) is presented by G.S.Altshuler from the Soviet Union and his studying team, as a complete methodological and theoretical system for inventive problem solving, which can accelerate the process of invention and obtain the productions of good quality ${ }^{[17]}$. The flow of TRIZ application can concluded to solve the inventive problem as figure 1: Firstly, We can use TRIZ, FBS (Function-Behavior-Structure) and other ways to represent engineering problem and transform it into a general problem; And then, the general solution is obtained by using the inventive tools.; Finally, the designer transforms the general solution into a 
particular solution with the help of analogy, as well as his own domain knowledge and design experience.

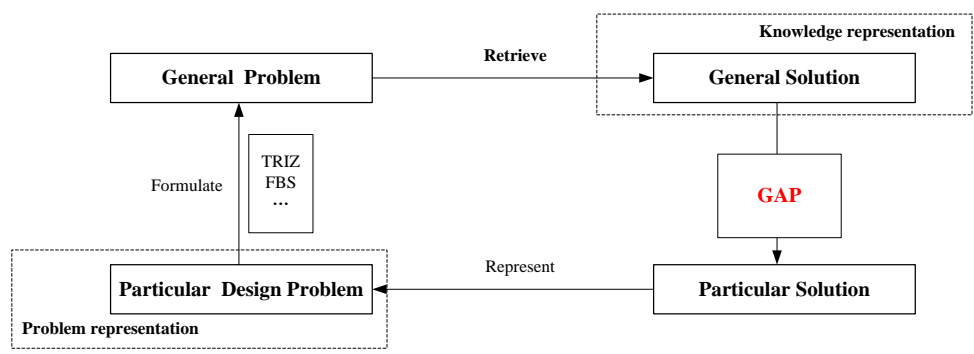

Figure1: The Flow of TRIZ Application

The general solution is not the final solution to the problem, providing the designer with a new possible approach to solve the problem. The general solution is a highly abstract. A gap exists between general solution and particular solution. The transformation process to a particular solution still requires the immense amount of knowledge from different practical domains, but the designer himself cannot grasp all the supporting knowledge required for solving the problem, which results in the lack of knowledge and enhances the difficulty to the inventive design.

As shown in figure 2, in order to help designers to get over the "gap" between the general solution and particular solution, we propose a multi-perspective and multilevel based inventive problem solving model. In this model, the design task is represented from 4 perspectives based on human comprehension in processing problems of product inventive design, including "what do you want to design? "(Considering inventive problem in a visual perspective, referring to specific design objects , with certain limitations, easy to cause mindsets, difficult to acquire creative solutions.), "What is it used for" (Considering the ultimate goal of design, representing the problem as the overall goal. By completely eliminating the existing production system limitations, describing the inventive problem from the functional level.), "How to achieve it?" (Thinking about the implementation of this design problem, transforming the design principles into concrete operations.) and "Which problem do you have?"(Considering the current problems as the driving force for the transformation between problem levels, including technical principles and design thinking, and five abstract attributes corresponding to domain information, functions, behavior-flow, scientific effects, and invention principles.) Designers could reconstruct a new problem representation by this way. 


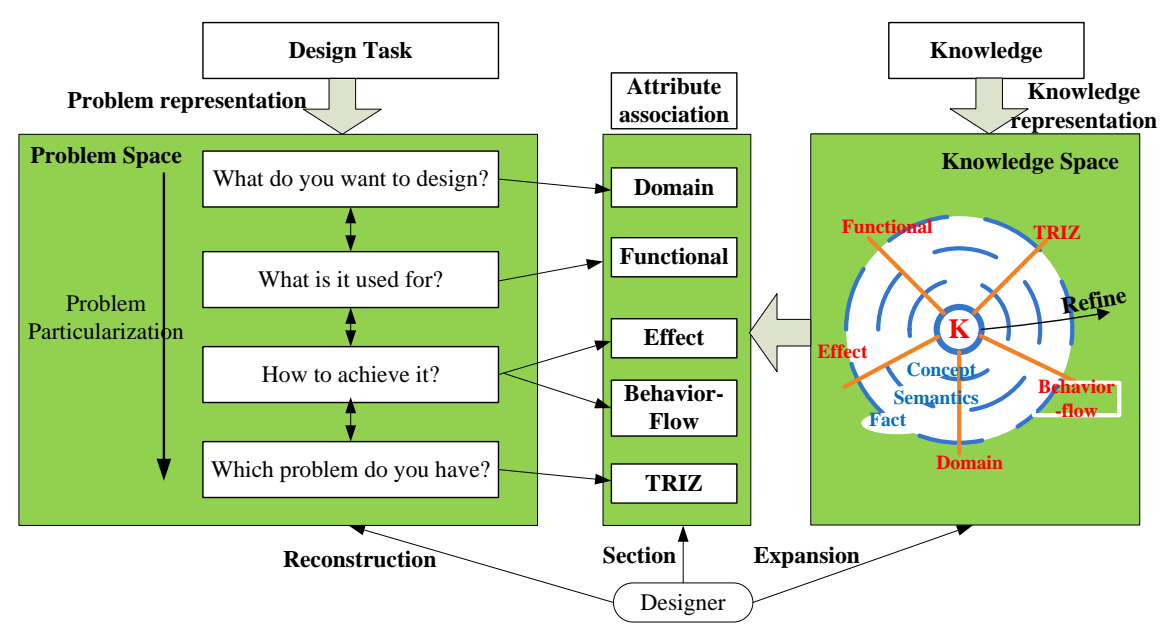

Figure 2: A multi-perspective and multi-level based inventive problem solving model

Besides, we extract five attributes from design knowledge-TRIZ, function, behavior-flow, effect, and domain attribute. An attribute is regarded as a description perspective and a retrieval perspective of knowledge. Each attribute is described from three levels from abstract to specific: concept, semantics and factual resources, which forms a multi-perspective and multi-level local knowledge base. Designers could choose any perspective and any level to understand the knowledge.

Finally, according to the relationship between attributes, we construct the semantic ontology of attributes. Relevant knowledge is searched and mined from patent database and network resources. Through semantic similarity calculation, knowledge within domain, in close domain and in further domain are provided for inventive design. Supporting designers to analyze and apply knowledge from different perspectives as well as at different levels is beneficial to motivating designers to make analogy and the complete knowledge transfer, thus facilitating the transforming process of general solutions to particular solutions.

\section{The organization and representation of design knowledge}

\subsection{The computer-oriented knowledge organization}

According to the different types of knowledge during the product inventive design process, we here classify the attributes of product design knowledge by TRIZ attribute, function attribute, behavior-flow attribute, effect attribute, and domain attribute, so as to fill the "gap" between the general solution and the particular solution. Meanwhile, each attribute can be described by different levels knowledge. Each level corresponds to different abstract layers, including conceptual layer, semantic layer and factual resource layer, we can thereby establish a multi-perspective design knowledge organization and representation model. As the figure shown in figure 3: 


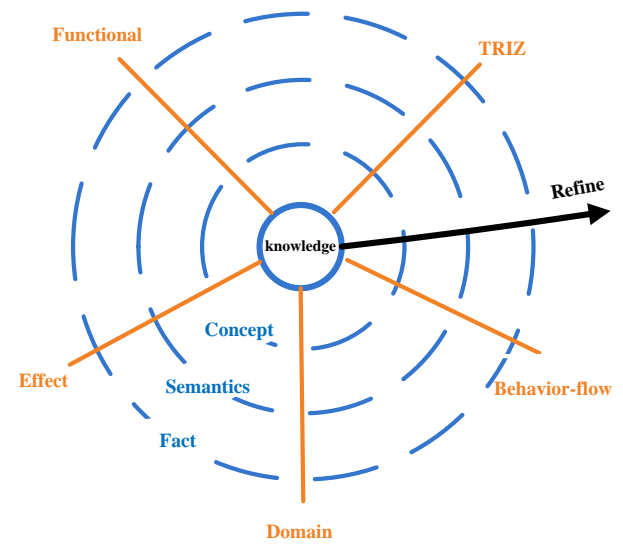

Figure 3: A multi-perspective and multi-level based knowledge representation model

Conceptual layer knowledge organization: The conceptual layer is a highly abstract representation of attributes and also the basic unit of the attribute description. A concept may contain many sub-concepts and may even be a sub-concept of another concept. A set of concept can form a grain, so that a multi-level attribute concept description is formed with the organization of the concept layer. The granularity conceptual structure of each attribute can be expressed in the following paper.

TRIZ attribute: It describes and expresses the principle, the general solution and the technological evolution rule of TRIZ, as shown in the figure 4:

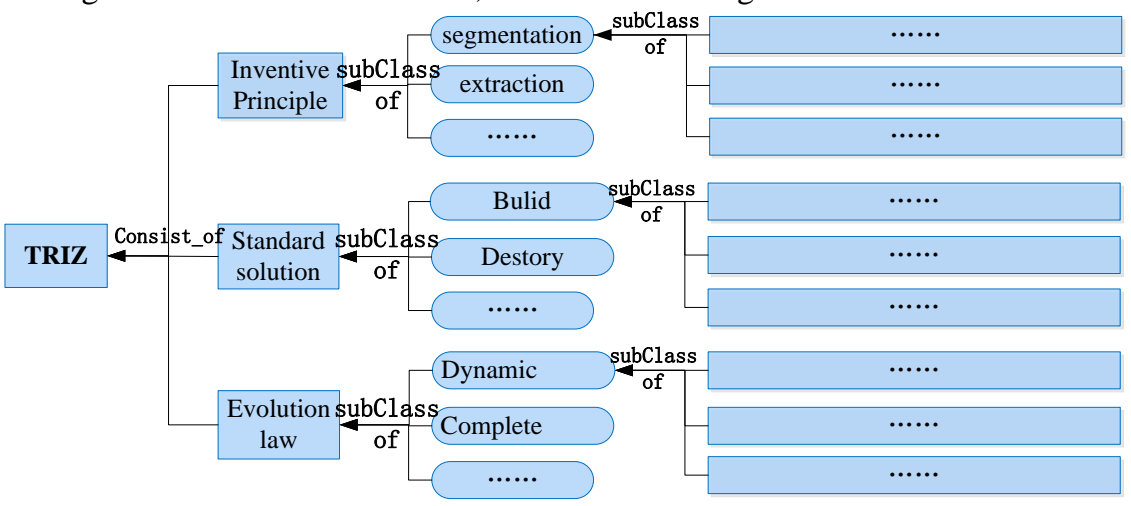

Figure 4 the conceptual layer of TRIZ attribute

Semantic layer knowledge organization: The semantic layer is a description of attribute content and the relationship between semantics, including attributedescription and attribute-association. Attribute is a description of the characteristics, which is meanwhile a concrete expression of attribute characteristics of knowledge, and it is convenient for designers to understand quickly knowledge and to establish the relationship between attributes by that between semantics. 
Factual layer knowledge organization: Facts refer to the detailed descriptions of practical case. Resources refer to resource contents obtained from attribute semantic description and Internet information expansion, including patent knowledge and extended knowledge of network resources within domain, in close domain, and in further domain.

Through the attribute concept and ontology association, we can retrieve the external network resources, and then the semantic similarity between the search result document and the attribute description is calculated to sort and to classify the searched results due to the knowledge of within domain, the close domain and the further domain. The vectorization of the text can be expressed as:

$$
d_{j}=\left(w_{1 j}, w_{2 j}, \ldots, w_{n j}\right)
$$

Where $d_{j}$ represents the document $\mathrm{j}, w_{i j}$ represents the weight value of the word $\mathrm{t}_{\mathrm{i}}$, and the weight of a word is calculated using TF-IDF (word reverse document frequency). The principle of TF-IDF is that the more frequently a word appears in a document, the greater weight value it has; The less common a word is, the greater its weight value is , i.e., words have a good distinguishing ability. The formula for calculating TF-IDF is as follows:

$$
w_{i j}=\frac{f_{i j}}{\max \left\{f_{1 j}, f_{2 j}, \ldots, f_{n j}\right\}} * \log \frac{N}{d f_{i}}
$$

Here $f_{i j}$ is the number of times the word $t_{i}$ appears in the document $d_{j}, \mathrm{~N}$ is the total number of documents, and $d_{f i}$ is the number of words $t_{i}$ in the document. The similarity between the document $\mathrm{q}$ and the document $d_{j}$ can be calculated as follows:

$$
\operatorname{sim}\left(d_{j}, q\right)=\frac{\sum_{i=1}^{n} w_{i j} \times w_{i q}}{\sqrt{\sum_{i=1}^{n} w_{i j}^{2}} \times \sqrt{\sum_{i=1}^{n} w_{i q}^{2}}}
$$

(Note: the similarity value is within $(0,1))$

While $\operatorname{sim} \in[0.7,1)$, the document $d_{j}$ belongs to within domain of the document q. While $\operatorname{sim} \in[0.4,0.7)$, the document $d_{j}$ belongs to close domain of the document $\mathrm{q}$. While, $\operatorname{sim} \in[0,0.4)$, the document $d_{j}$ belongs to further domain of the document q. The problem solving is a process of cooperation between relevant knowledge, and it causes easily mind-set relying on one single domain, which holds back the generation of new ideas. In this Internet era, the connection between local knowledge basis and internet resource should be well established during the organization and representation of knowledge, which can help the knowledge extension, as well as the designer's knowledge space expansion.

\subsection{The user-oriented knowledge representation}

The design knowledge representation contributes not only to computer retrieve and storage, but also to users' reading comprehension, so that it stimulates efficiently associative thinking. Based on the analysis above, we propose here one card-type multi-perspective and multi-level design knowledge representation template. The traditional cases, patents and other knowledge are divided into relatively independent 
parts according to different attributes, and each part is displayed in the form of card. Each card describes the knowledge in the conceptual, semantic and factual layers of each attribute. Designers can start reading knowledge from any card forming a multiperspective and multi-level knowledge structure, which helps understanding and knowledge transfer. In addition, the knowledge is marked by multiple attributes to form a structured text, which is beneficial for computer processing. An example of a design knowledge representation template shown in Figure 5.

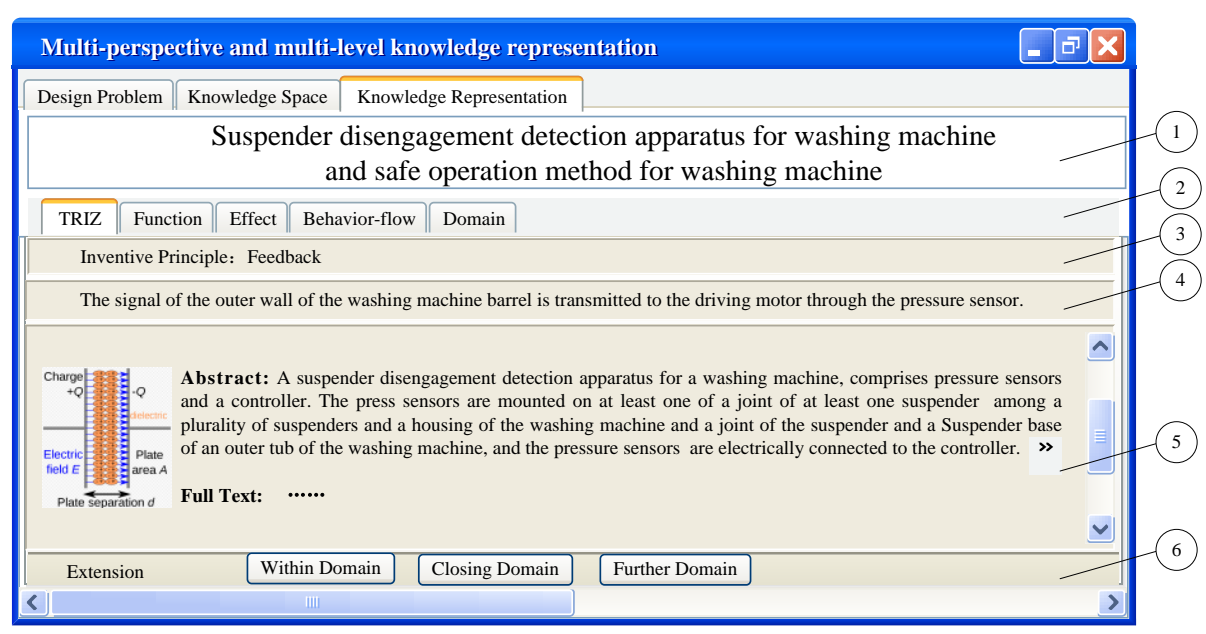

Figure 5: Knowledge representation template

Where, 1 is the title bar, displaying the name of the knowledge item; 2 is the attribute tab bar, including TRIZ, functions, effects, behavior flow and domain items. Designers are free to choose any attribute tag for browsing and reading; 3, 4, 5 are respectively the conceptual layer, the semantic layer and the factual layer of attributes; 6 are the extension layer of knowledge item, referring to semantic similarity algorithm, with which knowledge can be retrieved within domain, in close and in further domain. The user retrieves knowledge through the attribute tag, selects the content of interest in the knowledge space, and browses the knowledge item. With the knowledge representation of card-type, the user does not need to read the full text, and can even still understand the contents from different perspectives, thereby reducing the cognitive load of users, as well as improving the efficiency of knowledge application.

\section{Case study}

As a mature householdappliance, the washing machineistakenas an innovativedesignexampleinthepaper to show the application process of the above model. 


\subsection{Problem representation}

As shown in figure 6, the design task can be described as "Please use your imagination to provide an innovative design for the washing machine". There is relatively few restricted conditions, which is easy for designer to consider the problem from any angles.

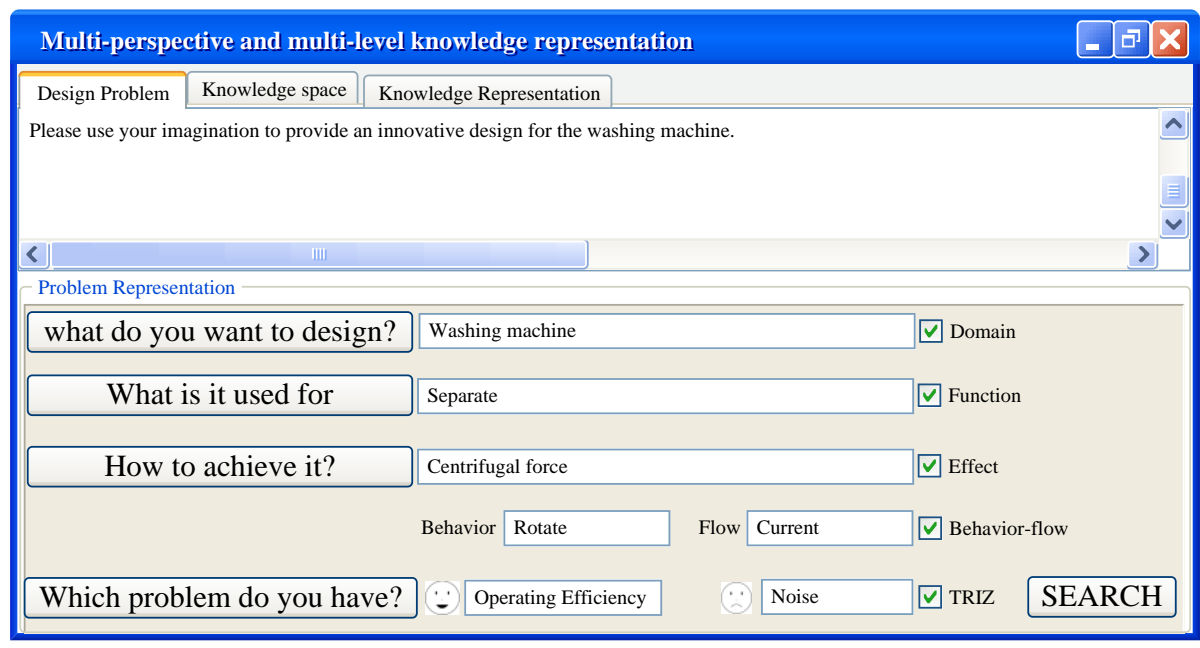

Figure 6:the interface of problem representation

i. Problem representation from"domain" perspective: Designers can type "Washing Machines" in "What are you design?" Input box to retrieve relevant knowledge so as to understand the current development and main problems of "Washing Machines".

ii. Problem representation form "functional" perspective: The main function of the washing machine is to clean the stains on the clothes, so the functional basis can be expressed as "separate/remove solid". Therefore, type "separate" in input box"what is used for?", to get all the patent knowledgeimplementing the "separation" function.

iii. Problem representation from "effect" perspective:Currently, the washing machine can be mainly divided intothe pulsator washing machine and the drum washing machine. Both of them are applied to reach the function of the product realized by "centrifugal force".

iv. Problem representation from"behavior-flow" perspective: Nowadays, the washing machine drives the engine to rotate by electric current, and generates centrifugal force to separate the stain from the clothes. In input box "how to achieve it?", we can enter "rotation-current" to get a case of using "centrifugal force" to achieve product function.

v. Problem representation from "TRIZ"perspective:The existing washing machine consists of a box body, an engine, a base and the other components. Thus it is very cumbersome and occupiesquite a large area. So it is only suitable for household 
usage. But while the people are traveling, they also need an equipment to help them to clean their clothes. The adaptability and the volume of washing machine form a set of technical contradictions. In input box "which problem do you have?" we can input "adaptability" into the harmful factors and put "the volume of stationary object" into the favorable factors to retrieve the knowledge to solve this contradiction.

Withthese five perspectives above to characterize the design problem, it can assist the designer to fully understand the design problem and find a breakthrough. The designer can select any one or several of the attributes to combine multiple knowledge for retrieve. In this paper, all these five perspectives are settled as examples.

\subsection{Knowledge representation}

Through the analysis above, we got the retrieved patent knowledge shown in figure 7 .

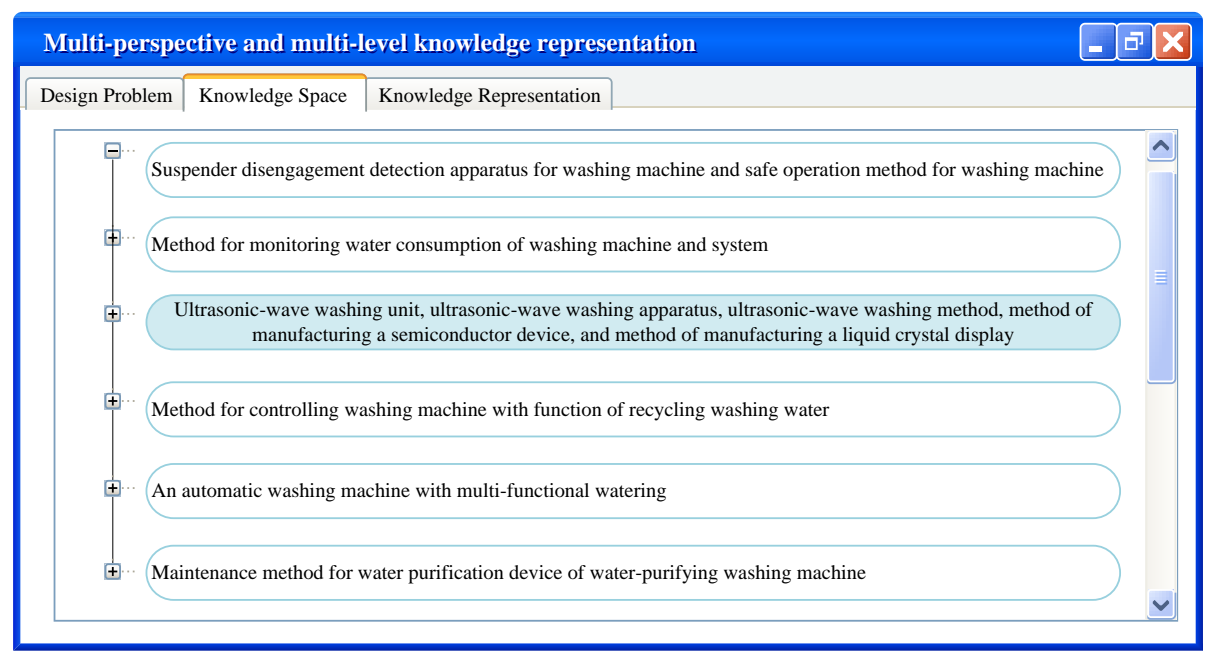

Figure 7: the interface of knowledge space

We choose one of the knowledge items to browse. As shown in figure 8, we can get knowledge from five perspectives. This is an ultrasonic cleaning unit with a sound wave at a frequency exceeding $20 \mathrm{kHz}$ beyond the human hearing range. The dirt layer is dispersed, emulsified, and stripped to reach to the cleaning purposes by direct and indirect effects on liquids and dirt from cavitations, and acceleration. At present, ultrasonic cleaning machines can be used in the machinery industry, the pharmaceutical industry, and the textile industry, etc. 


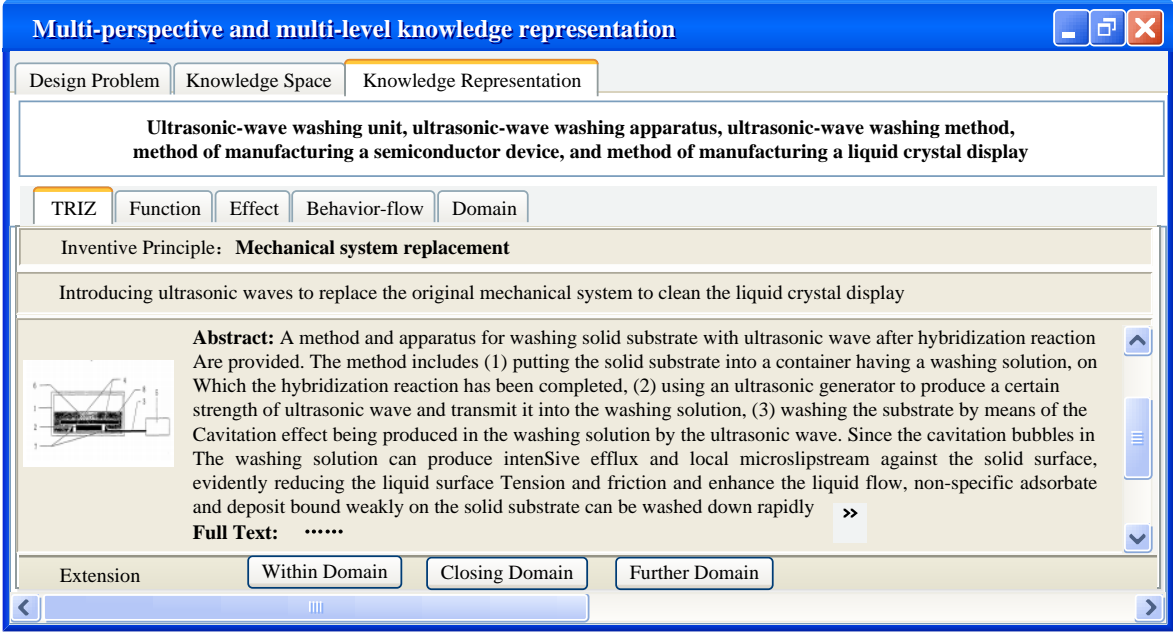

Figure 8: the interface of knowledge representation

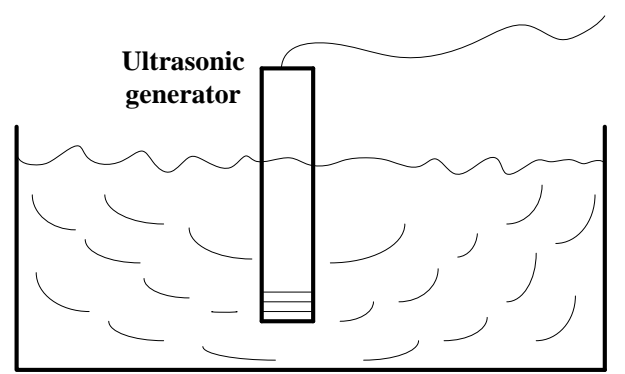

Figure 9: the conceptual solution

Based on the above, we have got a conceptual solution to design an ultrasonic washing machine. The conceptual solution is shown as in the figure 9.An ultrasonic generator is applied replacing the rotary pulse generator. In addition, the ultrasonic device is compact, and a $20 \mathrm{kHz}$ ultrasonic generator device has a size of $160 \mathrm{~mm} * 100 \mathrm{~mm} * 120 \mathrm{~mm}$, which is easy to carry for users in their backpacks. When it needs to wash clothes, just fill the pool with water and clothes, then put the ultrasonic generator into it. And it meanwhile solves the contradiction between "operational efficiency" and "noise".

\section{Conclusion}

Aiming at the transforming from the general solution to the particular solution during inventive problem solving, this paper proposes a multi-perspective and multi-level knowledge representation method from the perspective of human cognition and knowledge transfer, and provides knowledge to different abstraction degrees for the designer during the product design process, in order to compensate for the lack of 
knowledge between the general solution and the particular solution. And finally, it assists the designer to make association and analogy, so as to quickly and effectively propose innovative design solutions in the conceptual design stage.

Due to the problem characteristics and the required knowledge types at different stages, TRIZ principles, functions, behavior flow, effects, and domains are applied as attributes classification. Each attribute can be described in conceptual layer, semantic layer and factual layer, which forms a multi-perspective and multi-level local knowledge base. Moreover, it provides the designers with multi-level knowledge within domain, in close domain and in further domain, which is convenient for the designer to read and understand. Focusing on the current knowledge, it generates new knowledge on different levels, so as to stimulate the designers to make associative and analogical thinking. Besides, through problem representation from multi-attributes perspective, it allows designers to observe, analyze, and solve problems on different conceptual levels. The retrieval results given in the multi-perspective steps can give rise to different possible answers for the same design problem. In the future work, we will set up a general algorithm for the model and that it will support the difficulty of creating the global proposal network for all the possible answers.

\section{Acknowledgements}

This work is supported by the National Natural Science Research Foundation of China (NSFC) (No. 51435011) and the China Scholarship Council (CSC).

\section{Reference}

1. Lutters, E., van Houten, F. J., Bernard, A., Mermoz, E., \& Schutte, C. S.: Tools and techniques for product design. CIRP Annals 63(2), 607-630(2014).

2. Horvath, Imre.: A treatise on order in engineering design research.Research in Engineering Design 15(3), 155-181(2004).

3. Chandrasegaran, S. K., Ramani, K., Sriram, R. D., Horváth, I., Bernard, A., Harik, R. F., \& Gao, W.: The evolution, challenges, and future of knowledge representation in product design systems. Computer-aided design45(2), 204228 (2013).

4. Cong H,Tong L H.: Grouping of TRIZ inventive principles to facilitate automatic patent classification. Expert Systems with Applications34(1):788795(2008).

5. Prickett P, AParicio I.: The development of a modified TRIZ technical system ontology. Computers in Industry63(3):252-264(2012).

6. Yu H,Fan D.: Man-made boards technology trends based on TRIZ evolution theory. Physics Procedia33(6):221-227(2012).

7. Park H,Ree J J,Kim K.: Identification of promising patents for technology transfers using TRIZ evolution trends. Expert Systems with Applications 40(2):736-743(2013). 
8. Zhang, Y., Luo, X., Li, J., \&Buis, J. J.: A semantic representation model for design rationale of products. Advanced Engineering Informatics27(1): 1326(2013).

9. Qin, H., Wang, H., \& Johnson, A. L.: A RFBSE model for capturing engineers' useful knowledge and experience during the design process. Robotics and Computer-Integrated Manufacturing44, 30-43 (2017).

10. Aulive Homepage, http://www.aulive.com/, last accessed 2019/07/10.

11. Pro/innovator Homepage http://www.iwint.com/en/products/pro_innovator-20081208-211930.html, last accessed 2019/05/02.

12. PIXSEB Homepage, http://pixseb.com/, last accessed 2019/05/02.

13. CREAP Homepage, http://www.creap.cn/, last accessed 2019/05/02.

14. CREAX Homepage, https://www.creax.com/en/create/product-innovation, lasr accessed 2019/07/10.

15. Long-fan, LIU., Yan,LI., Jin-long, MA, Xiaojiao, DU., \& Hongwei, LIU.: Application of TRIZ in creative design based on knowledge granularity. Journal of Mechanical Engineering52(5), 22-32 (2016).

16. Cross, N.: Designerly ways of knowing. Springer, London (2006).

17. Yan, W., Liu, H., Zanni-Merk, C., Cavallucci, D. : IngeniousTRIZ: An automatic ontology-based system for solving inventive problems. KnowledgeBased Systems75, 52-65 (2015). 